\title{
Aerodynamic Characteristics of a Micro Multi-Rotor Aircraft with 12 Rotors Considering the Horizontal Wind Disturbance
}

\author{
Yao Lei ${ }^{1,2, * \mathbb{D}}$, Wenjie Yang ${ }^{1}$ and Hengda Wang ${ }^{1}$ \\ 1 School of Mechanical Engineering and Automation, Fuzhou University, Fuzhou 350116, China; \\ ly030312101@126.com (W.Y.); hengda_wang@163.com (H.W.) \\ 2 Key Laboratory of Fluid Power and Intelligent Electro-Hydraulic Control (Fuzhou University), \\ Fujian Province University, Fuzhou 350116, China \\ * Correspondence: yaolei@fzu.edu.cn; Tel.: +86-0591-22866791
}

Received: 16 September 2020; Accepted: 19 October 2020; Published: 21 October 2020

\begin{abstract}
Wind disturbance posed difficulties for the stability of the micro air vehicles (MAVs) with attitude variation. In this paper, the aerodynamic performance of a MAV with six coaxial rotor pairs considering the horizontal wind is investigated by both experiments and numerical simulations. First, the effect of the horizontal wind on the multi-rotor aircraft is analyzed in detail. Then, low-speed wind tunnel tests were performed to obtain the thrust and power consumption and the aerodynamic performance of the multi-rotor aircraft $(l / D=1.2$ and $h / D=0.19)$ with the rotational speed of $1500-2300 \mathrm{r} / \mathrm{min}$ in the horizontal wind ranged from 0 to $5 \mathrm{~m} / \mathrm{s}$. Finally, the distribution of streamline, the pressure of the blade tip, and the velocity and the vortices in the flow field of a multi-rotor aircraft with horizontal wind disturbance, were simulated and studied using the computational fluid dynamics (CFD) method. Through the comparison of experimental results and simulation results, it can be seen that the horizontal wind disturbance will increase power consumption to weaken the aerodynamic performance at higher rotor speeds. However, larger thrust and better hover performance are obtained at lower rotational speeds with good wind resistance. Additionally, due to the mutual induction between rotor wakes, the interactions of downwash flows become more intense at higher rotational speeds or larger wind speeds where the vortexes at the blade tip deformed and moved along with the wind.
\end{abstract}

Keywords: multi-rotor; coaxial rotor pairs; horizontal wind; wind tunnel tests; numerical simulation

\section{Introduction}

Recently, micro multi-rotor aircraft have developed rapidly due to the continuous improvement of technology in flight control. Micro multi-rotor aircraft have been widely employed in military and civil fields due to their simple configuration, high safety and convenient operation [1-5]. Compared with the traditional rotorcraft, they have a large increase in payload due to the increase in the number of rotors. In particular, multi-rotor aircraft with coaxial rotor pairs show better hover performance. However, there is strong aerodynamic interference between the adjacent coaxial rotor pairs, especially the upper rotors and lower rotors. Obviously, the existence of those aerodynamic disturbances will seriously affect the aerodynamic performance of micro multi-rotor aircraft [6,7]. In addition, they will be affected by wind gusts when a micro air vehicle is working outdoors, resulting in more complicated aerodynamic interference between rotors. Thus, it is necessary to study the ability of resisting wind disturbance for micro multi-rotor aircraft to improve their flight efficiency and aerodynamic characteristics.

Recent studies involved with aerodynamic performance are only focused on coaxial rotors. Deng et al. [8] analyzed the aerodynamic characteristics of coaxial rotors at a high-speed both in 
hover and forward flight with the wind tunnel test and numerical method. Lakshminarayan et al. [9] obtained the flow field distribution characteristics of coaxial rotors in hover with the computational fluid dynamic (CFD) method. Tan et al. [10] proposed an unsteady aerodynamic analysis to analyze the aerodynamic interference between coaxial rotors with a vortex particle method. Lei et al. [11,12] studied the aerodynamic performance of a coaxial rotor system with different rotor spacing and wind speed by experiments and the CFD method. Multiblock grids and the Multiple Frame of Reference (MFR) method were adopted by Jiang et al. for calculating hover characteristics of the coaxial rotor system [13]. In their studies on the aerodynamic characteristics of micro multi-rotor aircraft, Vikram et al. [14] studied the vortex formation, turbulent regimes, wake region and tip vortex formulation of the multi-rotor aircraft with various wind conditions using the CFD method. Zheng et al. [15] analyzed the characteristics of the downwash airflow for six-rotor UAVs in hover. Hwang et al. [16] studied the wake interaction between the rotors of a quadrotor on the hover and forward flight. Zhao et al. [17] studied the influence of airflow disturbance and rotor disturbance on Hex-rotor aircraft by designing an inversion sliding mode controller. Through the above studies, it can be found that the effect of the wind disturbance is not considered in the aerodynamic performance yet. However, the wind effect is not ignorable during the flight tests. Therefore, it is crucial to study the wind effect on aerodynamic characteristics of the multi-rotor aircraft to improve the manipulations in the flight control.

Considering that the wind effect on the aerodynamics of MAVs is undocumented without much reference, it is a challenge to lead concreteness. To be clear, the main research highlights are summarized as follows: (1) 12 rotors with six pairs of coaxial rotors are promoted to maximize the payload of the vehicle. It has a much wider class than those traditionally used, including the visible results from numerical simulations and higher accuracy from the experimental setup to demonstrate the validity of the present optimization method. (2) Wind effects on the thrust and power of multi rotor pairs are tested to demonstrate that additional thrust with the optimal configuration achieved a good wind resistance. This paper goes further by introducing the effect of wind disturbance as part of the engineering design and shows that it satisfies the design constraints whilst optimizing the combined electric and aerodynamic efficiency for typical hover conditions. Eventually, the feasibility of the proposed design is demonstrated through simulations and wind tunnel tests.

\section{Analysis of Theoretical Model}

The multi-rotor aircraft tends to have greater load capacity with more rotors. The structure of the multi-rotor aircraft studied in this paper is shown in Figure 1.


Figure 1. Structural sketch of a multi-rotor aircraft.

It can be seen that there are 6 coaxial rotor pairs with a total of 12 rotors. For each coaxial rotor pair, the upper and lower rotor rotate in opposite directions, and the rotational direction between adjacent rotors are also opposite. Although coaxial rotors were proven to have a simplified structure and better aerodynamic characteristics, the rotor interference including upper rotor and lower rotor, and also the adjacent rotor pairs, will pose a serious impact on their stability. In particular, the wind disturbance is introduced in this case. Figure 2 shows the airflow of a coaxial rotor pair without wind disturbance. It is shown that the downwash flows of the upper rotor are completely immersed in the inflow of the 
lower rotor when the wind is not introduced. On the contrary, the aerodynamic environment is much more complicated with the influence of crosswind, which is shown in Figure 3.

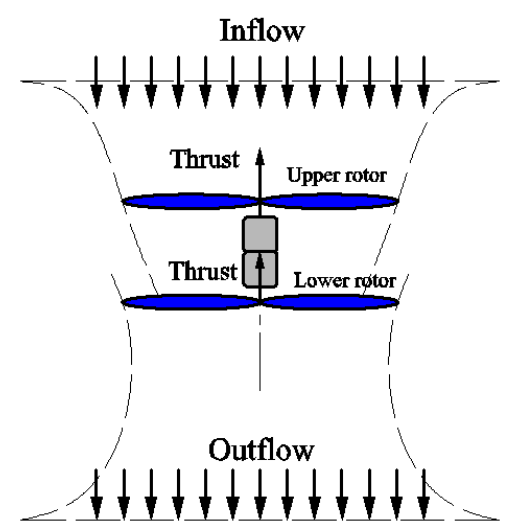

Figure 2. Direction of airflow a coaxial rotor pair without wind disturbance.



Figure 3. Aerodynamic model of adjacent coaxial rotor pairs with wind disturbance.

As shown in Figure 3, the downwash flows of coaxial rotor pairs moved with the horizontal wind and interfered with each other. In addition, with the disturbance of horizontal wind, each rotor of the multi-rotor aircraft will generate additional thrust $T_{w i}(i=1,2, \ldots, 12)$, and the total thrust $T_{i}$ of each rotor can be expressed as [18]:

$$
T_{i}=T_{r i}+T_{w i}=2 \rho A V V_{i}
$$

where $T_{r i}=k \Omega_{i}{ }^{2}$ is the thrust generated by the motor of rotor, $k$ is the proportionality coefficient. $\Omega_{i}, \rho$, $A, V_{i}$ and $V$ are the rotational speed of rotor, the density of air, the area of rotor disk, the extra induced speed and the total induced speed, respectively. The total induced speed of rotor is given by:

$$
V=\left[\left(V_{w} \cos \varphi+V_{i}\right)^{2}+\left(V_{w} \sin \varphi\right)^{2}\right]^{\frac{1}{2}}
$$

where $V_{w}$ is the wind speed, $\varphi$ is the angle between the axis of rotor and the horizontal crosswind. In this paper, $\varphi=90 \mathrm{deg}$ to characterize the horizontal wind. In addition, $V_{w}=0, V=V_{i}, T_{w i}=0$, and $T_{i}=T_{r i}$ when there is no wind disturbance.

Generally, the aerodynamic performance of a multi-rotor aircraft is characterized by the thrust coefficient $C_{T}$, power coefficient $C_{P}$ and hover efficiency $\eta$ :

$$
\begin{aligned}
& C_{T}=\frac{T}{\rho A \Omega^{2} R^{2}} \\
& C_{P}=\frac{P}{\rho A \Omega^{3} R^{3}}
\end{aligned}
$$




$$
\eta=\frac{C_{T}^{3 / 2}}{\sqrt{2} C_{P}}=\frac{T^{3 / 2}}{P \sqrt{2 \rho A}}
$$

where $R$ is the rotor radius.

\section{Wind Tunnel Tests}

\subsection{Experimental Setup}

The sketch of experimental setup is indicated in Figure 4.

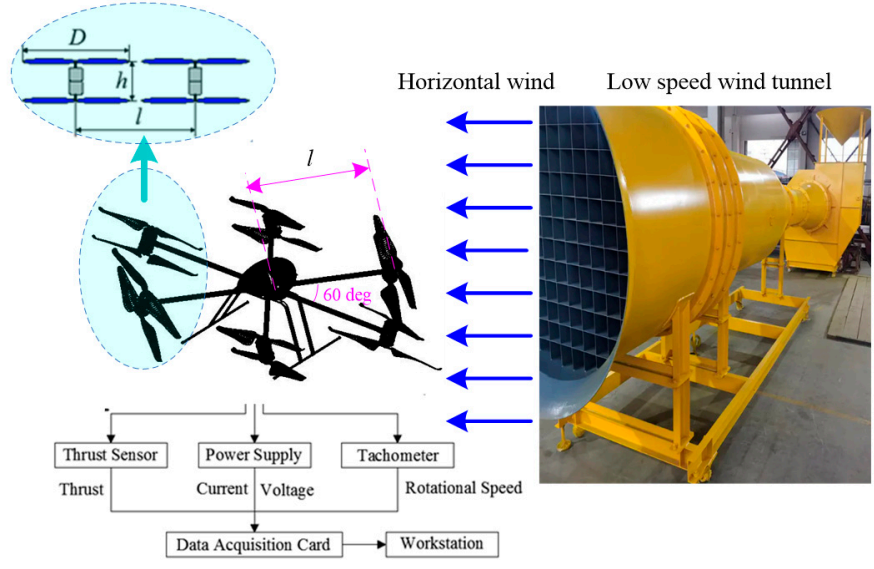

Figure 4. Sketch of experimental setup.

In Figure $4, D, h$ and $l$ are the diameter of the rotor, the distance between the upper and lower rotor of a coaxial pair and the distance between the rotational center of adjacent coaxial rotor pairs, respectively. Propellers are specially made with unidirectional carbon fiber fabrics with $15.7 \mathrm{~cm}$ of pitch and $2.8 \mathrm{~cm}$ of chord at 75\% position. The BLDC motor with KV 4800 is applied for each rotor, which is powered by lithium batteries with a capacity of 10,000 $\mathrm{mAh}$. The test section of the low speed wind tunnel is $3 \mathrm{~m}$ (length) $\times 3 \mathrm{~m}$ (width) $\times 2.5 \mathrm{~m}$ (height) to ensure sufficient space for maneuvering. A settling chamber is attached before the test section to characterize the output wind. Considering that the multi-rotor aircraft is generally disturbed by light air $(0.3-1.5 \mathrm{~m} / \mathrm{s})$, light breeze $(1.6-3.3 \mathrm{~m} / \mathrm{s})$ and gentle breeze (3.4-5.4 m/s) in the natural environment [19], the averaged wind speeds of $2.5 \mathrm{~m} / \mathrm{s}$ and $4.0 \mathrm{~m} / \mathrm{s}$ are applied in the low-speed wind tunnel. In the tests, the thrust was measured by a thrust sensor (model: CZL605, accuracy: 0.02\% F.S), and the power was obtained by collecting the voltage and current. All these data were recorded in real-time.

The experimental parameters are presented in Table 1.

Table 1. Experimental parameters.

\begin{tabular}{cccccc}
\hline Wind Speed $(\mathrm{m} / \mathbf{s})$ & Rotor Speed $(\mathbf{r} / \mathbf{m i n})$ & Diameter $(\mathbf{m m})$ & $h / D$ & $l / D$ & Rotor Pitch $(\mathbf{m m})$ \\
\hline $0,2.5,4.0$ & $1500 \sim 2300$ & 400 & 0.19 & 1.2 & 157 \\
\hline
\end{tabular}

\subsection{Experimental Results}

The variation of thrust and power consumption is shown in Figure 5. 




Figure 5. Thrust and power consumption in different rotational speeds.

It showed that both the thrust and power of the multi-rotor aircraft increased with the rotational speed. Compared with $0 \mathrm{~m} / \mathrm{s}$, the thrust decreased with the effect of horizontal wind with a higher rotor speed. However, the power still increased at the same time. This extra power may be caused by the aerodynamic interference between the upper and lower rotors of the coaxial rotor pair, as well as the strengthened wake between adjacent coaxial rotor pairs.

The variations of the increments of thrust coefficient $C_{T}$ and power coefficient $C_{P}$ are shown in Figure 6.

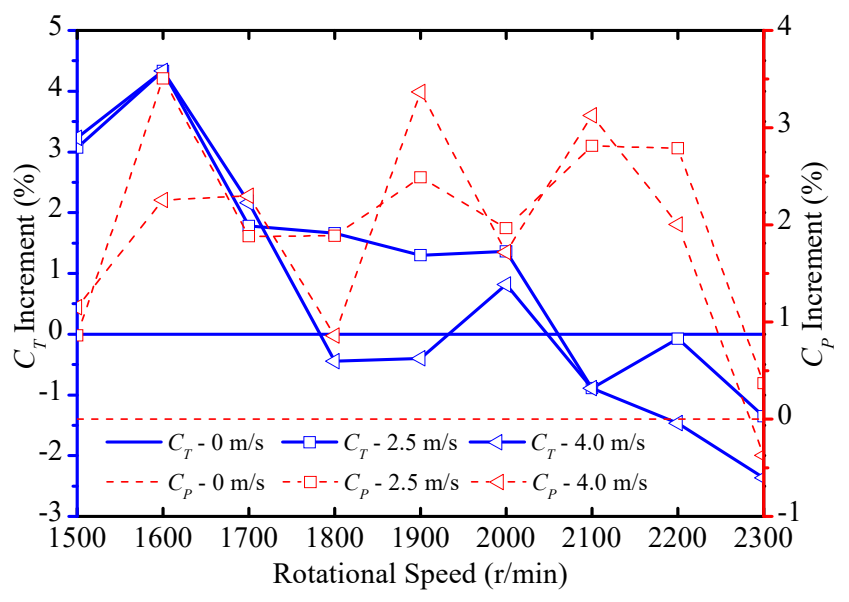

Figure 6. $C_{T}$ and $C_{P}$ increment with different wind speeds.

As shown in Figure 6, the thrust increment of $C_{T}$ gradually decreased with the rotor speed. In particular, $C_{T}$ with wind effect is lower than that at $0 \mathrm{~m} / \mathrm{s}$ from $2000 \mathrm{r} / \mathrm{min}$. On the contrary, the power coefficient $C_{P}$ at the horizontal wind of $2.5 \mathrm{~m} / \mathrm{s}$ and $4 \mathrm{~m} / \mathrm{s}$ increased significantly compared with $0 \mathrm{~m} / \mathrm{s}$, and the maximum increments were $3.51 \%$ at $2.5 \mathrm{~m} / \mathrm{s}$ with $1600 \mathrm{r} / \mathrm{min}$ and $3.37 \%$ at $4.0 \mathrm{~m} / \mathrm{s}$ with $1900 \mathrm{r} / \mathrm{min}$, respectively. This may have been caused by the strong interaction on downwash flows of adjacent coaxial rotor pairs.

The variation of hover efficiency $\eta$ is shown in Figure 7. 


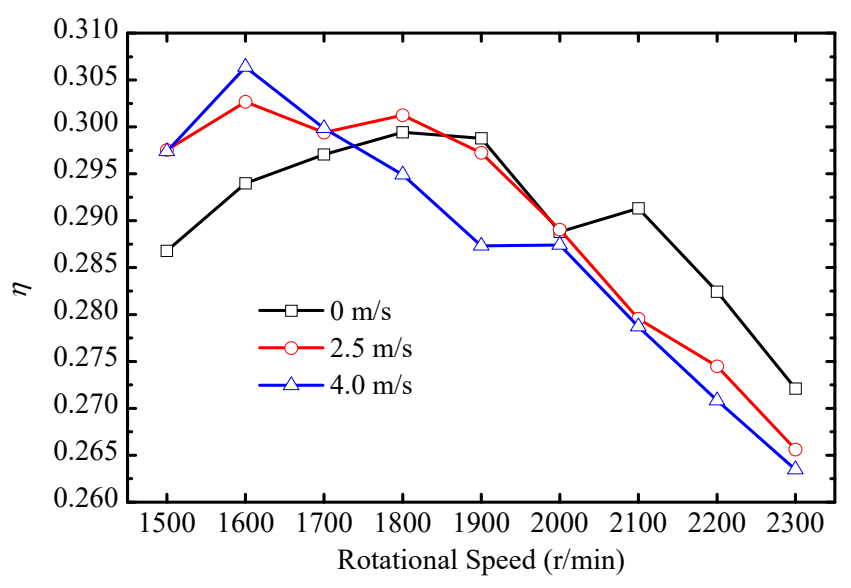

Figure 7. Hover efficiency $\eta$ at different wind speeds.

It is clear that there was higher hover efficiency with horizontal wind disturbance, with a lower rotor speed of around $1800 \mathrm{r} / \mathrm{min}$ compared with $0 \mathrm{~m} / \mathrm{s}$. The hover efficiency $\eta$ at the wind speed of $2.5 \mathrm{~m} / \mathrm{s}$ and $4.0 \mathrm{~m} / \mathrm{s}$ reached a maximum of 0.302 and 0.306 at $1600 \mathrm{r} / \mathrm{min}$. However, $\eta$ with wind effect decreased at higher rotor speed around $1800 \mathrm{r} / \mathrm{min}$. In addition, the hover efficiency without wind disturbance is obviously higher than that with wind disturbance. Moreover, lower hover efficiency is found at higher wind speed. This indicated that the horizontal wind disturbance strengthened the strong aerodynamic interference between the adjacent coaxial rotor pairs with a negative impact on the hover performance of the multi-rotor aircraft at higher rotational speed.

Therefore, although the multi-rotor aircraft consumes more power with the horizontal wind disturbance, the horizontal wind improved its aerodynamic performance by increasing hover efficiency at lower rotational speed. With the increase in the rotor speed, the performance decreased with higher power where the horizontal wind aggravated the downwash flows of the coaxial rotors.

\section{Numerical Simulations}

\subsection{Simulation Setup}

The flow field of multi-rotor aircraft can be simulated by CFD with ANSYS 18 to obtain the aerodynamic interference between rotors. For simulation setup, the Spalart-Allmaras (S-A) model was selected with the Pressure-Implicit with Splitting of Operators (PISO) algorithm and the Semi-Implicit Method. Additionally, second-order upwind is applied to obtain the momentum, energy equation, and turbulent viscosity. The mesh distribution with 6.87 million cells is shown in Figure 8.

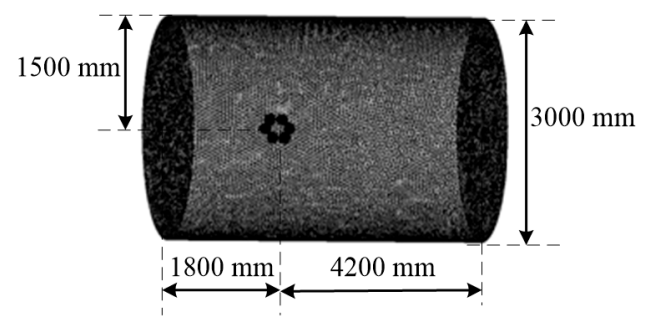

(a)



(b)

Figure 8. Mesh distribution: (a) Computational domain; (b) mesh details. 


\subsection{Simulation Results}

A comparison of thrust coefficient $C_{T}$ and power coefficient $C_{P}$ both in experiment and simulation is shown in Figure 9 to validate the reliability of the CFD simulation. It can be seen that the results of the experiment and simulation are basically consistent, and the CFD simulation is capable to capture the flow details.

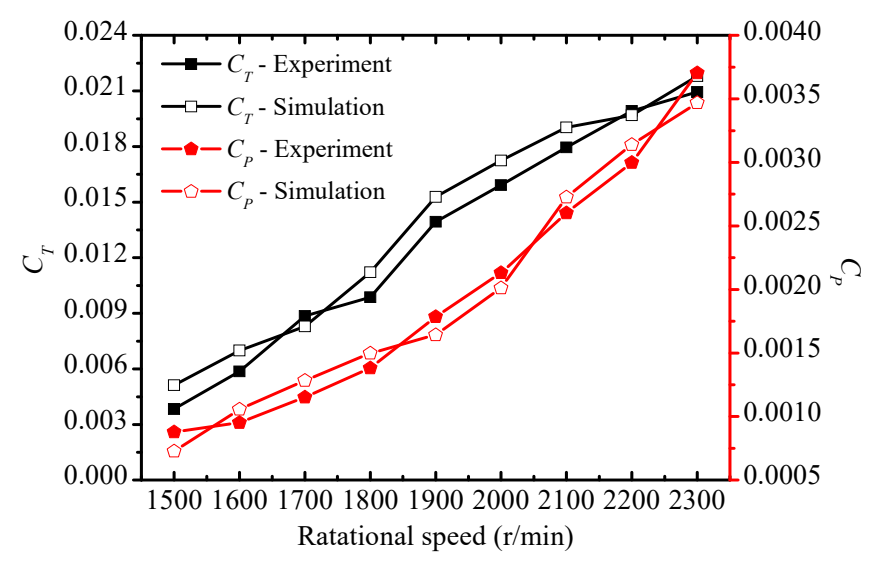

Figure 9. Comparison of $C_{T}$ and $C_{P}$ in experiment and simulation.

Streamline distributions of the multi-rotor aircraft are shown in Figure 10.

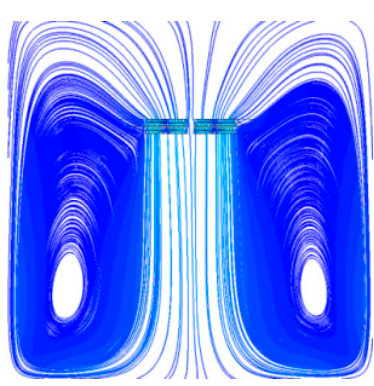

(a)

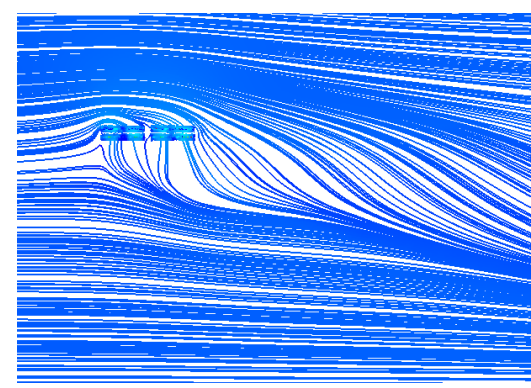

(b)

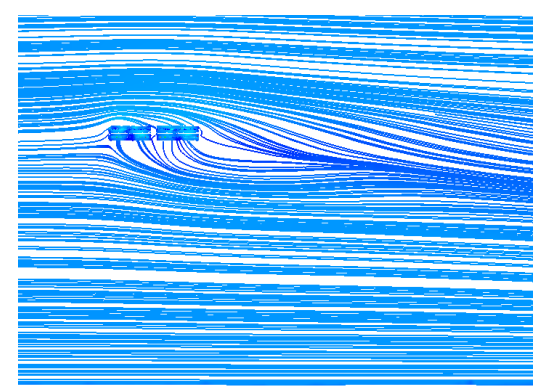

(c)

Figure 10. Streamline distribution: (a) $0 \mathrm{~m} / \mathrm{s}$; (b) $2.5 \mathrm{~m} / \mathrm{s}$; (c) $4.0 \mathrm{~m} / \mathrm{s}$.

As shown in Figure 10, the streamlines are symmetrically distributed where vortexes are formed below the blade tip when there is no wind disturbance. However, the regular vortexes below the blade tip disappear when the horizontal wind is introduced. Compared with the symmetrical streamlines without the effect of horizontal wind, the horizontal airflow directly acts on the rotor disk and flows to the lower right of rotors after being accelerated by the coaxial rotor pairs. Further, with the increase in wind speed, the slipstream moves more with the wind and the interaction between adjacent coaxial rotor pairs is also more intense. This may be the reason that the multi-rotor aircraft have larger power consumption with the effects of horizontal wind, which cause a decrease in hover efficiency.

The pressure distribution of blade tip of a coaxial rotor pair with various wind speeds is shown in Figure 11. 


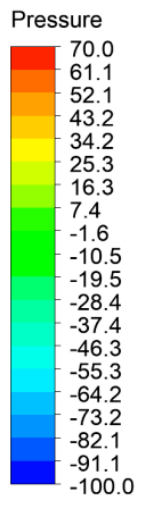

$[\mathrm{Pa}]$

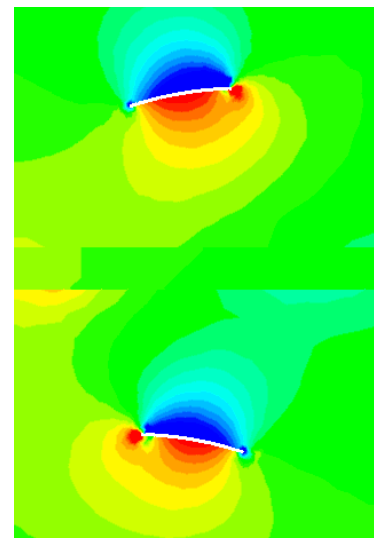

(a)

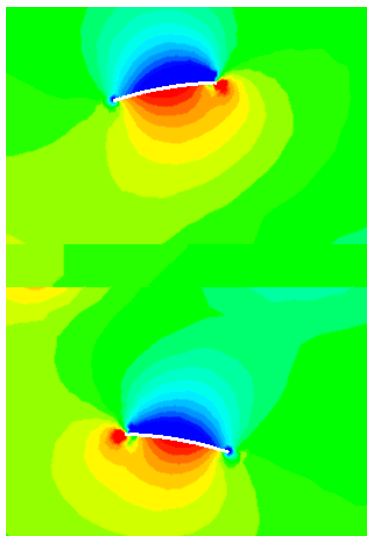

(b)



(c)

Figure 11. Pressure distribution of blade tip: (a) $0 \mathrm{~m} / \mathrm{s}$; (b) $2.5 \mathrm{~m} / \mathrm{s}$; (c) $4.0 \mathrm{~m} / \mathrm{s}$.

As shown in Figure 11, the positive pressure is concentrated on the lower surface of the rotor, and the negative pressure is mainly concentrated on the upper surface of the rotor. Compared with $0 \mathrm{~m} / \mathrm{s}$, the area of positive pressure and negative pressure are enlarged to some extent with the effect of horizontal wind, which may result in an increase in the thrust of the rotor. In addition, the rotors have a larger pressure difference at $2.5 \mathrm{~m} / \mathrm{s}$ than that at $4.0 \mathrm{~m} / \mathrm{s}$. Combined with experimental results, this may explain why that the multi-rotor aircraft has higher thrust coefficient and better aerodynamic performance at $2.5 \mathrm{~m} / \mathrm{s}$ than those at $4.0 \mathrm{~m} / \mathrm{s}$.

The velocity distribution of adjacent coaxial rotor pairs is shown in Figure 12.
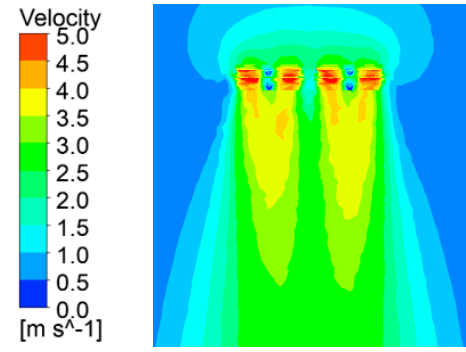

(a)



(b)

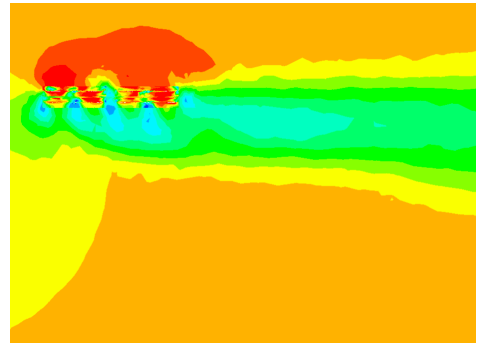

(c)

Figure 12. Velocity distribution: (a) $0 \mathrm{~m} / \mathrm{s}$; (b) $2.5 \mathrm{~m} / \mathrm{s}$; (c) $4.0 \mathrm{~m} / \mathrm{s}$.

As shown in Figure 12, it is clear that the downwash flows are symmetrically distributed below the rotors when there is no wind disturbance. When the horizontal wind is introduced, the interaction between the downwash flows is strengthened and the downwash flow move with the wind, which may consume more power and reduce the aerodynamic performance of the multi-rotor aircraft. However, the interference between the rotors of a coaxial rotor pair is weakened with the horizontal wind, which may cause larger thrust on the rotors. Further, as the horizontal wind speed increases, the aerodynamic interaction between the adjacent coaxial rotor pairs will be more obvious and stronger.

The vortices distributions of the multi-rotor aircraft with different wind speeds are shown in Figure 13. 


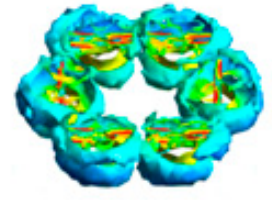

(a)

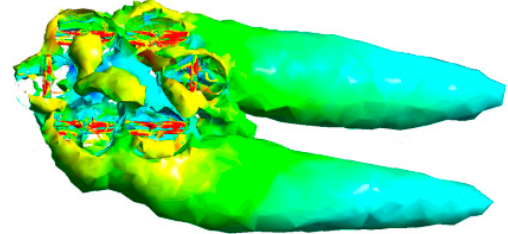

(b)

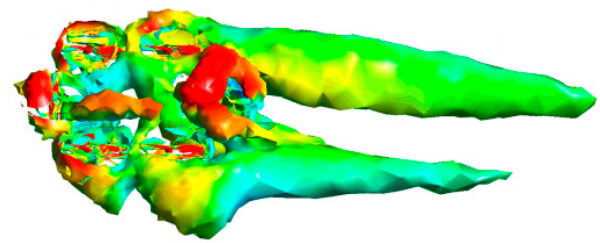

(c)

Figure 13. Vortices distribution: (a) $0 \mathrm{~m} / \mathrm{s}$; (b) $2.5 \mathrm{~m} / \mathrm{s}$; (c) $4.0 \mathrm{~m} / \mathrm{s}$.

It can be seen that vortices interference is much stronger with horizontal wind disturbance compared with no wind disturbance. It will eventually lead to vortex deformation. Additionally, the trailing vortex of the front rotors may be immersed with the inflow of the rear rotors, which will cause strong disturbance with the rear rotors. In the end, it may result in an increase in power consumption. With the increase in wind speed, the rotor interference is more intense, which may even interact with the inflow of upper rotor and waken the hover performance dramatically.

\section{Conclusions}

In this paper, the horizontal wind effect on aircraft with 12 rotors is studied both with wind tunnel tests and CFD simulation. Conclusions are as follows:

(1) The power consumption of the multi-rotor aircraft obviously increases with the horizontal wind speed. However, compared with no wind disturbance, the thrust is also increased at the rotational speed of 1500-1800 r/min where the aerodynamic performance is much improved. This indicated that the multi-rotor aircraft can effectively resist the horizontal wind disturbance at lower rotational speeds.

(2) Compared with no wind disturbance, the pressure difference on the rotor surface with the influence of horizontal wind is obviously enlarged, which brings more thrust for rotors. In addition, vortices formed at the blade tip, and dissipated with the horizontal wind, resulting in deformation, which has a negative effect on the hover efficiency of the multi-rotor aircraft.

(3) Rotor interference between the adjacent coaxial rotor pairs is strengthened with the horizontal wind disturbance. The downwash flows of the rotors moved in the direction of the horizontal wind, which causes that the wakes of the front rotors have serious effects on that of the rear rotors. Further, as the wind speed increases, this aerodynamic interference becomes more intense. Future study will attempt to conduct a series of flight tests that attempt to obtain an aerodynamic model considering the rotor interference to amend the conventional control theory.

Author Contributions: Y.L. carried out experiments; W.Y. revised the manuscript and performed new set of simulations. H.W. wrote the manuscript with the assistance of Y.L. All authors have read and agreed to the published version of the manuscript.

Funding: This research was supported by the National Natural Science Foundation of China (Grant No. 51505087), Fuzhou University Jinjiang Science and Education Park (No. 2019-JJFDKY-59) and Fujian Provincial Industrial Robot Basic Components Technology Research and Development Center (2014H2004).

Acknowledgments: The authors thank the Key Laboratory of Fluid Power and Intelligent Electro-Hydraulic Control (Fuzhou University), Fujian Province University, and Fuzhou University Jinjiang Science and Education Park for applying the experimental field.

Conflicts of Interest: The authors declare no conflict of interest.

\section{References}

1. Salazar, S.; Romero, H.; Lozano, R.; Castillo, P. Modeling and Real-Time Stabilization of an Aircraft Having Eight Rotors. J. Intell. Robot. Syst. 2009, 54, 445-470. [CrossRef]

2. Teng, H.; Ahmad, I.; Alamgir, M.S.M. 3D Optimal Surveillance Trajectory Planning for Multiple UAVs by Using Particle Swarm Optimization with Surveillance Area Priority. IEEE Access 2020, 18, 86316-86327. [CrossRef] 
3. Kim, J.; Gadsden, S.A.; Wilkerson, S.A. A Comprehensive Survey of Control Strategies for Autonomous Quadrotors. Can. J. Elect. Comput. E 2020, 43, 3-16.

4. Rodriguez-Ramos, A.; Sampedro, C.; Bavle, H. A Deep Reinforcement Learning Strategy for UAV Autonomous Landing on a Moving Platform. J. Intell. Robot Syst. 2019, 93, 351-366. [CrossRef]

5. Esakki, B.; Ganesan, S.; Mathiyazhagan, S. Design of Amphibious Vehicle for Unmanned Mission in Water Quality Monitoring Using Internet of Things. Sensors 2018, 18, 3318. [CrossRef] [PubMed]

6. Ye, L.; Xu, G. Calculation on flow field and aerodynamic force of coaxial rotors in hover with CFD method. Acta Aerodynam. Sin. 2012, 30, 437-442.

7. Sunada, S.; Tanaka, K.; Kawashima, K. Maximization of thrust torque ratio of a coaxial rotor. J. Aircr. 2005, 42, 570-572. [CrossRef]

8. Deng, J.H.; Fan, F.; Liu, P.A. Aerodynamic characteristics of rigid coaxial rotor by wind tunnel test and numerical calculation. Chin. J. Aeronaut. 2019, 32, 568-576. [CrossRef]

9. Lakshminarayan, V.K.; Baeder, J.D. Computational investigation of micro-scale coaxial rotor aerodynamics in hover. J. Aircr. 2010, 47, 940-955. [CrossRef]

10. Tan, J.; Sun, Y.; Barakos, G.N. Unsteady loads for coaxial rotors in forward flight computed using a vortex particle method. Aeronut. J. 2018, 122, 693-714. [CrossRef]

11. Lei, Y.; Bai, Y.; Xu, Z.J.; Gao, Q.J. An experimental investigation on aerodynamic performance of a coaxial rotor system with different rotor spacing and wind speed. Exp. Therm. Fluid Sci. 2013, 44, 779-785. [CrossRef]

12. Lei, Y.; Lin, R.Z. Effect of wind disturbance on the aerodynamic performance of coaxial rotors during hovering. Meas. Control 2019, 52, 665-674. [CrossRef]

13. Jiang, Y.N.; Li, H.; Jia, H.G. Aerodynamics Optimization of a Ducted Coaxial Rotor in Forward Flight Using Orthogonal Test Design. Shock Vib. 2018, 2018, 2670439. [CrossRef] [PubMed]

14. Vikram, P.; Balasubramanian, E.; Manova, M. Aerodynamic Interaction Studies on Amphibious Vehicle in Forward and Hovering Flight. Int. J. Auto. Mech. Eng. 2019, 16, 7391-7412. [CrossRef]

15. Zheng, Y.J.; Yang, S.H.; Liu, X.X. The computational fluid dynamic modeling of downwash flow field for a six-rotor UAV. Front. Agric. Sci. Eng. 2018, 5, 159-167. [CrossRef]

16. Hwang, J.Y.; Jung, M.K.; Kwon, O.J. Numerical study of aerodynamic performance of a multirotor unmanned-aerial-vehicle configuration. J. Aircr. 2015, 52, 839-846. [CrossRef]

17. Zhao, C.J.; Bai, Y.; Gong, X. Hex-rotor unmanned aerial vehicle controller and its flight experiment with aerodynamic disturbance. Opt. Precis. Eng. 2015, 23, 1088-1095. [CrossRef]

18. Nguyen, D.H.; Liu, Y.; Mori, K. Experimental Study for Aerodynamic Performance of Quadrotor Helicopter. Trans. Jpn. Soc. Aeronaut Space Sci. 2018, 61, 29-39. [CrossRef]

19. Lei, Y.; Wang, H.D. Aerodynamic Optimization of a Micro Quadrotor Aircraft with Different Rotor Spacings in Hover. Appl. Sci. 2020, 10, 1272. [CrossRef]

Publisher's Note: MDPI stays neutral with regard to jurisdictional claims in published maps and institutional affiliations.

(C) 2020 by the authors. Licensee MDPI, Basel, Switzerland. This article is an open access article distributed under the terms and conditions of the Creative Commons Attribution (CC BY) license (http://creativecommons.org/licenses/by/4.0/). 